\title{
A procedure for the classification of eclipsing binaries
}

\author{
O. Yu. Malkov ${ }^{1,2,3,4}$, E. Oblak ${ }^{3}$, E. A. Avvakumova ${ }^{5}$, and J. Torra ${ }^{6}$
}

\author{
${ }^{1}$ Institute of Astronomy of the Russian Academy Sci. 48 Pyatnitskaya St., 119017 Moscow, Russia \\ e-mail: malkov@inasan.ru \\ 2 Department of Physics, Moscow State University, 119992 Moscow, Russia \\ 3 UMR 6091, Observatoire de Besançon - CNRS, 41 bis avenue de l'Observatoire, 25010 Besançon Cedex, France \\ 4 South African Astronomical Observatory, PO Box 9, Observatory 7935, South Africa \\ 5 Department of Physics, Ural State University, Lenin Street 51, 620083 Ekaterinburg, Russia \\ ${ }^{6}$ Departament d'Astronomia i Meteorologia, Universitat de Barcelona, Avda. Diagonal 647, 08028 Barcelona, Spain
}

Received 25 July 2006 / Accepted 12 January 2007

\section{ABSTRACT}

\begin{abstract}
A procedure for the automatic classification of eclipsing binaries is presented. The procedure is based on the data from 1029 classified systems and allows for the classification of a given system based on a set of observational parameters, even if the set is incomplete. The procedure is applied to six large surveys of eclipsing variables. About 5300 systems were classified for the first time and can be used for the determination of the astrophysical parameters of their components.
\end{abstract}

Key words. binaries: eclipsing - methods: data analysis - methods: statistical

\section{Introduction}

Eclipsing binaries are critically important for the determination of the fundamental properties of stars: masses, radii, temperatures and luminosities. They also provide critical tests of stellar physics, stellar evolution and stellar structure theories. More recently, eclipsing binaries have provided vital information on a wide variety of phenomena ranging from cosmology to exoplanets.

Independent stellar mass and luminosity determination is possible only for components of eclipsing binaries with the spectrum lines of the two components. However, they represent only some $5 \%$ of all known eclipsing binaries. So, for statistical investigations it is necessary to estimate at least approximate values of fundamental parameters (such as mass and radius) for eclipsing binaries with unknown spectroscopic elements.

The process of mass and other astrophysical parameter estimation differs for different evolutionary classes of systems. So, to properly estimate astrophysical parameters one needs to know the class of a given system.

A procedure for determination of the evolutionary class from observational data was first proposed by Svechnikov \& Snezhko (1974) and is still used in the General Catalogue of Variable Stars (1985-1988), hereafter GCVS. The procedure is based on a restricted number of systems with known classes contained in the old Svechnikov (1969) catalogue and, as our analysis shows (Malkov et al. 2005), is not accurate enough.

Useful ideas for classification of eclipsing binaries can be found also in a statistical study made by Giuricin et al. (1983). They mostly dealt with three classes of systems (detached, semi-detached and contact) and examined the statistics of the lightcurve morphologies, eclipse depths, periods and spectral types. So, Giuricin et al. (1983) have not considered the systems with unknown spectral classification, whereas spectra are often unknown for relatively faint, in general, eclipsing binaries. Only $22 \%$ of GCVS eclipsing binary spectra of primaries are known and there is no spectral classification in lists of eclipsing binaries obtained as by-products of microlensing surveys (see e.g. OGLE data for variable stars, Rucinski \& Maceroni 2001).

Thus, a more modern procedure is required that allows users to classify all eclipsing binaries based on a set of observational parameters, even if the set is incomplete.

A new catalogue of 6330 eclipsing variable stars was compiled by the authors (Malkov et al. 2005). The catalogue was developed from the GCVS and its text comments by including recently published information about classification of about 850 systems and making corresponding corrections of the GCVS data. About 200 classifications were recently added in the catalogue; thus it represents the largest list of eclipsing binaries classified from observations.

In the present study the catalogue has been used to develop the most comprehensive set of rules for the classification of eclipsing binaries to date, and to construct a procedure that allows users to classify eclipsing binaries based on a set of observational parameters, even if the set is incomplete.

The scheme of classification is presented in Sect. 2. A statistical analysis of the catalogue and a procedure for evolutionary class determination are given in Sect. 3. Results of the application of the procedure to various lists of eclipsing binaries are summarized in Sect. 4. In Sect. 5 we draw our conclusions.

\section{Classification scheme}

We distinguish the following classes of eclipsing binaries: detached (D), semi-detached (S) and contact (C) systems. Detached and contact systems have sub-classes in our notation as listed below and we use the second (and in some cases the third) letter for the sub-class designation. We do not separate various sub-classes of semi-detached systems, but for uniformity use a one-letter identification ( $\mathrm{S}$ ) for a system class, reserving the second position for a potential sub-class designation. 
The following classes of systems are considered here (see details in Malkov et al. 2005):

Main sequence systems (DM) - detached main sequence systems, where both components are main sequence stars that do not fill their inner Roche lobes.

Systems with two subgiants (DR) - detached systems with two subgiant components, where both stars do not fill their Roche lobe (alternative names: AR Lac systems, RS CVn systems, or long period RS CVn systems). The hotter component in such binaries usually has a spectral type of either F or $G$ and the systems show stronger emission lines in the spectrum outside eclipses. They typically are expected to have negligible mass exchange, although the majority of known RS CVn binaries are classified as detached in GCVS.

Giant and supergiant systems (DG) - systems with at least one member evolved well away from the main sequence (i.e., supergiant or late-type giant, e.g., VV Cep). Their evolutionary stage is generally unclear (Giuricin et al. 1983), but if the secondary component is a main sequence star, they are likely to be in a prior-to-mass exchange stage.

Semi-detached systems (S) - systems where the more massive component is the main sequence star and the less massive secondary component, which is usually the cooler and larger one, is a subgiant, which fills (or nearly fills) its Roche lobe.

Late-type contact systems (CW) - contact systems with the spectrum of the primary usually later than about F0 (also called W UMa systems). According to Binnendijk (1977), CW (classical W UMa-type contact) binaries can be divided into A-type (the larger star is hotter, the primary minimum is the transit) and W-type (the smaller star is hotter, the primary minimum is the occultation). Hereafter we designate them CWA and CWW systems, respectively, and we leave the $\mathrm{CW}$ code for W UMa-type binaries of unknown sub-class.

Early-type contact systems (CE) - contact systems of early spectra with both components close to their Roche lobes. The spectrum of the more massive component is usually not later than about F0 (Svechnikov 1969) or even A0 (Pribulla et al. 2003).

Near-contact systems (CB). This class was introduced by Svechnikov (1969) as being similar to W UMa-stars, where both components do not fill their Roche lobes and their physical characteristics are similar to those of $\mathrm{CW}$ systems. They are also called short-period RS CVn-type or W UMa-similar non-contact systems. Formally they can be referred to as detached or semidetached systems, but their lightcurves are peculiar and distorted by gaseous streams, and the brightness of the components is slightly variable, which makes them similar to contact systems of late spectral type. Pribulla et al. (2003) designate them as Btype contact systems (similar to the $\beta$ Lyr lightcurve), whose components are in physical but not in thermal contact. These are also sometimes called binaries with two stars of very different effective temperature enclosed in a common envelope with the secondary located below and to the left of the main sequence in the
HR diagram. According to Shaw (1994) we distinguish F-type near-contact binaries (the primary component is at or near the Roche lobe, the secondary component is inside the Roche lobe, the light curve is usually asymmetric) and V-type near-contact binaries (the primary component is inside the Roche lobe, the secondary component is at or near the Roche lobe, the light curve never shows asymmetry), respectively, CBF and CBV. CB is used for near-contact binaries of unknown sub-class.

We leave the $\mathrm{C}$ code for contact binaries of unknown sub-class.

\section{Statistical analysis}

The compiled catalogue is not completely inclusive or exclusive. However, the collected data allow us to make a preliminary statistical analysis and to find relations between different parameters for various classes of eclipsing binaries. Such an analysis is presented in this section.

The following data, contained in the Malkov et al. (2005) catalogue are considered here:

1. Depth of primary minimum, $A_{1}$, mag.

2. Depth of secondary minimum, $A_{2}$, mag.

3. Depth difference, $\mathrm{d} A=A_{1}-A_{2}$, mag.

4. Morphological type of the light curve (EA, EB, EW; as in the GCVS).

5. Period of the eclipsing variable star, $P$, day.

6. Information on the sign of period variability as follows:

d: derivative is negative (period decreases);

i: derivative is positive (period increases);

$\mathbf{v}$ : derivative is non-zero and the sign varies (period increases and decreases);

$\mathbf{u}$ : derivative is non-zero, but the sign is unknown (period increases or/and decreases).

7. Duration of primary eclipse, DI, phase.

8. Duration of secondary eclipse, DII, phase.

9. Phase of secondary minimum, MinII-MinI, phase.

10. Spectral type of the primary star, $\mathrm{Sp}_{1}$.

11. Luminosity class of the primary star.

12. Spectral type of the secondary star, $\mathrm{Sp}_{2}$.

13. Luminosity class of the secondary star.

\subsection{Depth of minima}

Let us consider eclipsing binary systems satisfying the following conditions:

- the system consists of two detached, non-elliptical components;

- there is no reflection effect in the system;

- there is no third source of light (third component, disc, stream, common envelope, etc.) in the system;

- the components are not variable;

- the system shows total eclipses.

In Fig. 1 we show results of theoretical considerations of the location of such systems in the $A_{1}-A_{2}$ plane, where $A_{1}$ and $A_{2}$ are depths of primary and secondary minimum, respectively.

Equal depths of minima (equal temperatures). Firstly, line A indicates the location of systems whose light curves have equal depths of primary and secondary minima, $A_{1}=A_{2}$ (the primary in such a system is $\mathrm{N}$ times larger and $\mathrm{N}^{2}$ times brighter than the secondary). By definition observational points cannot be situated to the left of that line. 


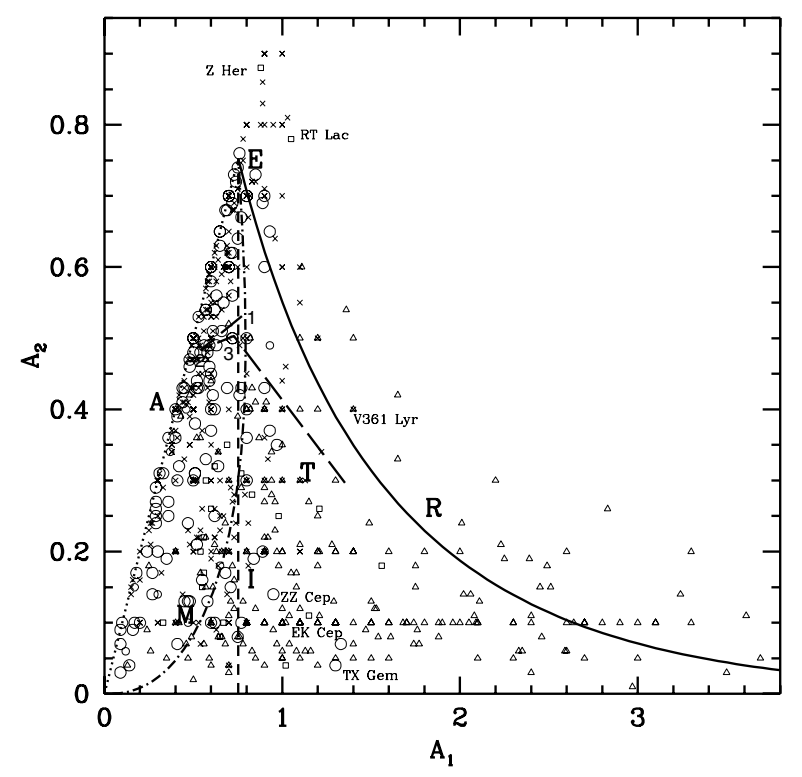

Fig. 1. Theoretical location of detached systems with total eclipses and catalogued systems in the $A_{1}$ (depth of primary minimum) $-A_{2}$ (depth of secondary minimum) plane. Line A - systems with components of equal temperatures, i.e., $A_{1}=A_{2}$. Line $\mathrm{R}-$ systems with components of equal radii, upper limit for detached systems. Line I systems with components of equal luminosities, i.e., $A_{1}=2.5 \log 2$, left limit for so called "inverse" systems (where brighter component is smaller). Line $\mathrm{M}-$ systems with $L_{2} / L_{1} \propto\left(R_{2} / R_{1}\right)^{5.5}$ (approximate relation for O9.5 - M0 MS stars). Line T - evolutionary track of a $2.8 m_{\odot}+$ $2.5 m_{\odot}$ system (see text for details). Point $\mathrm{E}-$ systems with identical components. Catalogued systems: circles - DM systems (larger), DG systems (smaller), squares - DR systems, triangles - $\mathrm{S}$ systems, crosses $-\mathrm{C}$ systems. The indicated systems are discussed in the text.

Equal radii. A depth of the $i$ th minimum of an eclipsing binary, satisfying the criteria listed at the beginning of this section, can be calculated as:

$A_{i}=-2.5 \log \left(\frac{L_{\min , i}}{L_{1}+L_{2}}\right)$,

where $L_{\min , i}$ is the luminosity of the system at the $i$ th minimum and the sum of components' luminositites $L_{1}+L_{2}$ is the luminosity of the system in maximum (out of eclipse). For systems with components of equal radii $L_{\min , 1}=L_{2}$ and $L_{\min , 2}=L_{1}$.

So, it can be shown that systems with $R_{1}=R_{2}$ are located on the line $\mathrm{R}$, satisfying the following equation:

$A_{2}^{R}=-2.5 \log \left(1-10^{-0.4 A_{1}}\right)$.

No observed system satisfying the listed conditions can be located above that line. Hereafter we ignore observational errors.

Systems with identical components $\left(T_{1}=T_{2}, R_{1}=R_{2}\right)$ are located at the point $\mathrm{E}$.

Brighter component is smaller than the fainter one. Let's call a system "inverse" when its brighter component (with luminosity $L_{1}$ ) is smaller than the fainter one (with luminosity $L_{2}$ ). Here occultation of the brighter component by the larger one is a primary minimum, when (assuming a total eclipse) integrated luminosity of the system is equal to luminosity of the fainter (larger) component $L_{2}$. By definition $L_{2}<L_{1}$. This means that the primary minimum here cannot be shallower than $A_{1}^{I}=2.5 \log 2 \approx 0.75$.
A vertical line I in Fig. 1, satisfying this equation, indicates the left border for the area of "inverse" systems and the location of systems with components of equal luminosities. So, if a system satisfies the listed conditions and has primary depth $A_{1}<A_{1}^{I}$, the brighter component is also the larger one. Line I does not serve as the right border for the area of "non-inverse" systems.

Main sequence components and post-MS evolution. It would be instructive to indicate also a place for systems with two MS components. Luminosities and radii of MS stars (at least from late $\mathrm{O}$ to early $\mathrm{M}$ ) satisfy quite well the following relation:

$L / L_{\odot} \propto\left(R / R_{\odot}\right)^{\alpha}$

where $\alpha=5.5$. If we consider a system with components of different radii, then, to a zeroth approximation:

$L_{\min , 1}=L_{2}+L_{1}\left(1-\frac{R_{2}^{2}}{R_{1}^{2}}\right)$

where $R_{1}$ is assumed to be larger than $R_{2}$, and $L_{1}>L_{2}$ (which is true for two MS components).

From Eqs. (1), (3) and (4) one can find the place occupied by systems with two MS components. It is indicated by line M in Fig. 1 and the equation for the line $\mathrm{M}$ can be written:

$A_{1}^{M}=-2.5 \log \left[1-\frac{t^{2 / \alpha}}{1+t}\right] ; \quad t=10^{0.4 A_{2}}-1$

Detached main sequence systems satisfying the listed conditions should not deviate significantly from the line M, i.e., for them

$\left|A_{1}-A_{1}^{M}\right| \leq \delta A_{1}$

where $\delta A_{1}$ is estimated to be about 0.3 (see discussion below).

During post-MS evolution, when components expand and mass transfer begins, systems leave the line M. An evolutionary track of a $2.8 m_{\odot}+2.5 m_{\odot}$ system is shown in Fig. 1 (Line T). The points indicated in the figure are: 1 - beginning of primary expansion, 2 - beginning of secondary expansion, 3 - beginning of mass transfer. Luminosities and radii of the components are drawn from Nelson \& Eggleton's 2001 calculation of algol type system evolution (see their Sect. 3, case AS - slow evolution).

The following conclusions can be drawn from Fig. 1 .

The detached configuration excludes a large depth of both minima and such systems cannot be situated above the line R (if one ignores observational errors). Detached systems with total eclipses and components of equal radii should lie at the line $\mathrm{R}$.

A detached system with total eclipses and $A_{1}<0.75$ (Line I) is not "inverse": its brighter component is larger.

Detached main sequence systems cannot be situated far to the right of the line $\mathrm{M}$; such systems should not deviate significantly from the line $\mathrm{M}$.

All these conclusions are valid for any photometric band and do not depend on the limb-darkening law.

We have considered system with total eclipses. It can be shown that a partial eclipse shifts a system in the $A_{1}-A_{2}$ plane toward the coordinate origin.

The catalogued systems on the $A_{1}-A_{2}$ plane are also shown in Fig. 1. 
Detached main sequence systems (DM). We have found that known DM systems on the $A_{1}-A_{2}$ plane satisfy the conditions listed in the previous section, i.e., do not lie above the line R, defined by Eq. (2) and do not deviate to the right of the line $\mathrm{M}$ by more than 0.3 . The three exceptions are EK Cep, TX Gem and ZZ Cep.

Marques et al. (2004) have recently confirmed the preMS nature of the low mass component of EK Cep. The enlarged (in comparison with the MS one) radius of the secondary leads to a shift of the system away from the DM-area on the $A_{1}-A_{2}$ plane.

Budding et al. (2004) have included TX Gem in their Catalogue of Algol Type Binaries, but they based this on the GCVS classifier and the latter is defined according to the old Svechnikov \& Snezhko (1974) procedure.

Cester et al. (1978) have indeed found ZZ Cep to be an anomalous (although distinctly detached) system with dimensions and luminosity of the secondary typical for a subgiant star more distant from the zero age main sequence than the primary.

So, the nature of TX Gem and ZZ Cep remains unclear.

The majority of the DM systems with total eclipses satisfy Eq. (6) and we estimate $\delta M$ to be about 0.3 . The exceptions are a few systems with approximately equal depths of primary and secondary minima (V760 Sco, SS Lac, V453 Cyg). They are early B-type pairs with similar components, so we conclude that Eq. (6) is valid for A- to K-type systems.

Detached systems with two subgiants (DR). In Fig. 1 almost all DR systems lie below the line R. Two exceptions are RT Lac and Z Her.

Popper \& Ulrich (1977) in their comprehensive study of RS CVn binaries note that Z Her is the only system in their list in which the more massive star is the less evolved. In all other cases the smaller (and hotter) component was less massive than the larger and cooler one.

Recently Çakirli et al. (2003) have found that the smaller and hotter component of RT Lac also has a larger mass than the other component.

So, we can assume that these two "inverse" DR systems are at a different stage of evolution than "classical" DR systems and that this stage is characterised by mass transfer. This can be the reason for such unusually high values of $A_{2}$ for RT Lac and Z Her.

Detached systems with a giant or supergiant (DG). There are 21 DG systems in the catalogue and $A_{2}$ values are known only for 8 of them. All of them satisfy the condition $A_{2} \leq A_{2}^{R}(2)$.

Here we make a natural assumption that systems with unknown $A_{2}$ are obviously low- $A_{2}$ systems. That is why we believe that the largest- $A_{1}$ systems $\mathrm{BL}$ Tel $\left(A_{1}=2 \mathrm{~m} \cdot 32, A_{2}\right.$ is unknown) and V381 Sco $\left(A_{1}=3.7, A_{2}\right.$ is unknown) satisfy the condition $A_{2} \leq A_{2}^{R}$ (2) and therefore really belong to DG.

Semi-detached systems (S) One interesting conclusion that can be drawn from Fig. 1 is that the $S$ systems avoid the vicinity of the point $\mathrm{E}$. The reason for this conclusion is the absence of observed algols with similar components: such pairs, being expanded, almost simultaneously evolve into the contact or nearcontact state, or after a short burst of thermal timescale mass transfer come to a long phase of nuclear timescale mass transfer, when luminosities and radii of the two components are already quite different.
Table 1. Limiting amplitudes and periods for the catalogued systems.

\begin{tabular}{llllll}
\hline \hline Class & $A_{1 \max }$ & $A_{2 \max }$ & $\mathrm{d} A_{\max }$ & $P_{\min }$ & $P_{\max }$ \\
\hline DM & 0.97 & 0.76 & 0.81 & 0.593 & 30.0 \\
DR & 1.56 & 0.63 & 1.38 & 1.983 & 39.3 \\
DG & 3.7 & 0.49 & $>3$ & 1.613 & 7430 \\
S & 4 & 0.6 & 3.64 & 0.309 & 38.5 \\
CB & 1.4 & 0.71 & 0.88 & 0.310 & 1.225 \\
CBF & 0.98 & 0.3 & 0.8 & 0.507 & 0.776 \\
CBV & 0.90 & 0.37 & 0.62 & 0.508 & 0.913 \\
CE & 0.89 & 0.86 & 0.28 & 0.500 & 1.886 \\
CWA & 0.71 & 0.60 & 0.13 & 0.261 & 0.820 \\
CWW & 0.94 & 0.90 & 0.3 & 0.220 & 0.469 \\
\hline
\end{tabular}

All of that poses an upper limit of $A_{2}$ for $\mathrm{S}$ systems. We estimate the limit to be around 0 . 6 . For semi-detached systems, the depth of secondary minimum does not exceed $A_{2}^{R}$ (see Eq. (2)) by more than 0.2 .

Contact systems. Photometric data in the GCVS. Analysing the depth of minima for contact systems, we have reduced magnitudes given in different photometric systems.

The GCVS is protometrically heterogeneous. Magnitudes in the GCVS are given in about 25 different photometric systems. To estimate upper/lower limits of observational photometric parameters, like the minimum depth, for a given class of systems, especially for contact binaries, we must properly reduce data from one system to another.

Photometry for $97 \%$ of the selected eclipsing binaries is given in one of the following four systems: $p$ (photographic), V (visual, photovisual, or Johnson's V), Hp (Hipparcos) and B (Johnson's B). In a first approximation we consider p and B as being equivalent and the Hipparcos magnitude as not differing much from V (Bessell 2000).

To statistically estimate the difference in $\mathrm{B}$ and $\mathrm{V}$ we have investigated some 40 light curves obtained in both magnitudes for 25 contact binaries (we have not faced the problem of reducing magnitudes analysing the depth of minima for detached and semi-detached systems) that have been published since late 1960 s to the present. We have found that for both amplitudes $A_{1}$ and $A_{2} A_{B} / A_{V}=1.07 \pm 0.01$. Below we always make estimations for limits of observational photometric parameters in $\mathrm{V}$, reducing $\mathrm{B}$ data from the GCVS correspondingly.

Catalogued contact systems. According to the results of comparison of observational data in different photometric bands discussed in the previous section, we obtain $A_{1}, A_{2}$ and $\mathrm{d} A=A_{1}-A_{2}$ upper limits for all classes of contact systems.

CB systems generally do not lie above the line R (2). CBF and CBV systems occupy approximately coincident areas on the $A_{1}-A_{2}$ plane. The small number of $\mathrm{CBF}$ and $\mathrm{CBV}$ systems in our catalogue prevents us from drawing more definite conclusions.

The majority of the CBF systems have $A_{1}<0$ m.98. The exception is V361 Lyr. Hilditch et al. (1997) noted a gross asymmetry in the light curves during the first half of the system cycle and they omitted the data between phases 0.10 and 0.48 in solving the light curves. The system requires further study.

The maximum values for $A_{1}, A_{2}$ and $\mathrm{d} A$ for the catalogued systems are given in Table 1. 


\subsection{Light curve morphological type}

As investigations show, morphological type EA can indicate any class of system except CW and CE. Type EB can stand for any class of system but DR. Morphological type EW is assigned to C systems only.

\subsection{Periods}

Limiting values of periods of the catalogued systems are summarized in Table 1.

The limits on the parameter space for CB systems are larger than the corresponding limits for $\mathrm{CBF}$ and $\mathrm{CBV}$ systems, while $\mathrm{CBF}$ and $\mathrm{CBV}$ systems occupy approximately the same area in the parameter space.

Some comments should be made regarding the period values of the following systems.

Exceptions to $\mathrm{CW}$ systems are the rather long period UZ Oct (CWA, $P=1$ d.149) and CV Cyg (CWW, $P=0.983)$, whose outstanding properties have been repeatedly mentioned (Lapasset \& Sistero 1984 and Vinko et al. 1996, respectively) but not yet explained.

Other extremely long period systems are the early-type LY Aur (CE, $P=4$ d.002) and V348 Car (CB, $P=5$ d.562). They were discussed in Stickland et al. (1994) and Hilditch \& Evans (1985), respectively, but require further study.

\subsection{Period changes}

Period changes are indicated in our catalogue for 385 systems, in particular for 246 systems with known classes. Hereafter in this section we will count only systems for which a period change is indicated.

Periods of 70 systems decrease (d). Among them are systems of almost all classes, including one short-period DG (WR+O) system CQ Cep (Antokhina et al. 1987), except CE and CBF classes. Periods of 74 systems increase (i). There is no CBV system among them. Periods of 49 systems have variable sign of change (v). Neither CE nor CBV systems belong to this group. Periods of 53 systems change, but the sign of that change is unknown (u). This group contains no CE and CBV system.

So, we can make the following conclusions concerning period variability as an indication of system class.

Periods of CE systems increase; this apparently indicates a mass transfer from the less massive component to the other, i.e., these systems have already passed a rapid phase of thermal timescale mass transfer and the initially primary component has a smaller mass than the other.

Periods of CBV systems decrease. This can indicate that the systems shrink due to mass transfer from the more massive primary undergoing Roche lobe overflow. We can expect to observe a reverse process (period increasing) in the CBF systems, but their statistics is too poor to make any definite conclusions.

Note that CE and CBV classes in our catalogue contain 16 and 10 systems, respectively. In both cases about half of the systems of a given class have no indication on period variability (that can mean either constancy of the period or lack of data for period variability determination). So we consider our conclusions as preliminary ones.

Period variations can arise, beside mass transfer, from the light-travel time effect from a tertiary companion in a system. It is also possible that periodic variations arise from the effects of a magnetic activity cycle.

\subsection{Phase of secondary minimum and duration of eclipses}

For 148 systems in the catalogue, the phase of secondary minimum is known. The phase MinII-MinI ranges from 0.262 to 0.797 .

MinII-MinI $>0.5$ occurs about two times more often than MinII-MinI $<0.5$ in the catalogue. It is supposed that there is an observer tendency for systems with equal or nearly equal depths of minima to designate primary and secondary minima so that the time between a primary and a subsequent secondary minima is larger than the time between a secondary and a subsequent primary minima. However, we failed to find a correlation between MinII-MinI and $\mathrm{d} A$.

For 62 of the 148 systems, the evolutionary class is known. Only DM systems show a significant difference in MinII-MinI from phase 0.5. The upper limit of this deviation for DR systems is found to be 0.04 . For other classes of systems, the secondary minimum occurs at phase $0.5 \pm 0.025$.

Further evidence for a non-circular orbit is a difference between duration of the primary and secondary eclipses (DI-DII).

DI-DII for catalogued systems is given only for the EA morphological type (i.e., $\mathrm{D}, \mathrm{S}$ and $\mathrm{CB}$ systems) and ranges from -0.08 to +0.08 .

$\mathrm{CB}$ systems have equal eclipse duration.

The upper limit of (DI-DII) modulus for DR systems is 0.02 .

The difference of eclipse durations can reach larger values for DM and S systems. This value varies from -0.08 to +0.02 for algols and such an anomaly of secondary minimum duration can be caused by a transit of an optically thick accretion disc rather than by an eccentric orbit.

Neither data on MinII-MinI (with two exceptions) nor data on DII are given in the GCVS for the catalogued DG systems. However, their large periods can in principle allow for large orbit eccentricities and, consequently, relatively large |DI-DII| and MinII-MinI values.

\subsection{Spectra}

The results on spectra of the catalogued systems are summarized in Fig. 2. The secondary spectrum is assumed to be always later than the primary one.

Detached systems. The spectral type of the hotter component in DM systems is usually O5.5 to G8 (two exceptions are M eclipsing binaries YY Gem and CM Dra) with the cool component being one and a half spectral type later at most. Exceptions are the bright system $\alpha \mathrm{CrB}(\mathrm{A} 0+\mathrm{G} 5)$ and the preMS system BM Ori (B2+A7). These limits come from observational selection effects: one can barely observe spectroscopically faint systems and secondaries with large magnitude difference.

Spectra of the catalogued detached systems with a giant or supergiant vary widely as well: from mid-O to early-M.

For DR systems the spectral type of the primary is F or G and the spectral type of the secondary varies quite insignificantly: from $\mathrm{G} 5$ to $\mathrm{K} 3$.

Semi-detached systems (S). Investigating $\mathrm{S}$ systems with known spectra of both components we have found that the spectral type of the hotter component is usually O5.5 to F9 and the cool component can be as late as K5.

Note however, two pairs with both components being $\mathrm{K}$ giants: RZ Cnc (K1III+K3-4III, Olson 1989) and AR Mon (K0III+K3III, Popper 1976). These two systems, together with 


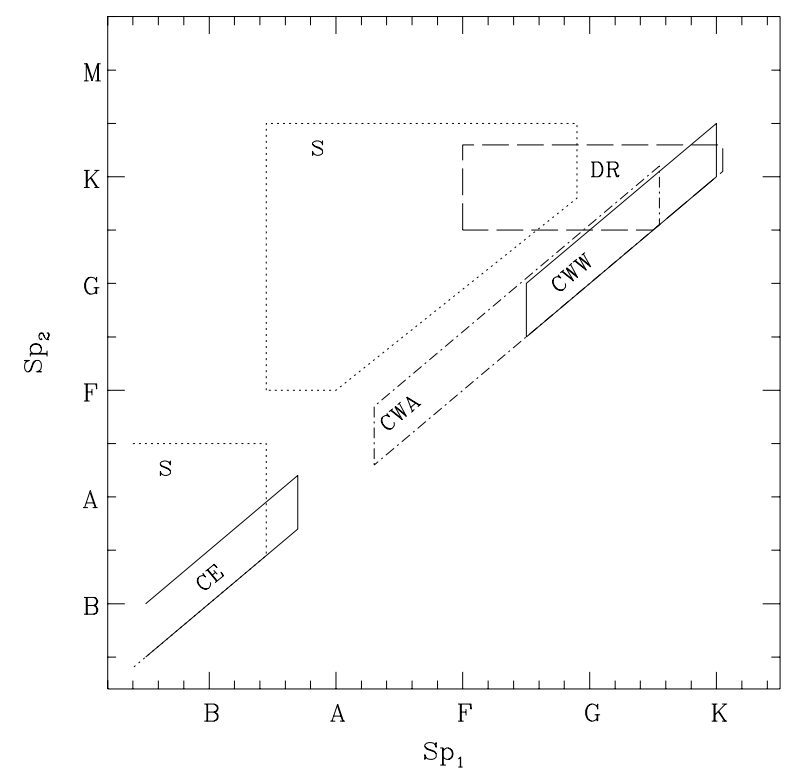

Fig. 2. Selected classes of systems in the $\mathrm{Sp}_{1}-\mathrm{Sp}_{2}$ plane. Spectra of $\mathrm{DM}$ and DG systems range from $\mathrm{O}$ to $\mathrm{M}$. Spectra of $\mathrm{CB}$ systems range from B to K. Cool semi-detached systems are not shown.

AD Cap and RV Lib, comprise Popper's (1980) group of cool semi-detached systems (we do not include in this group a DR system RT Lac with both components being subgiants and with depth of secondary minimum $A_{2}=0.78$ that is too large for a semi-detached system, as can be seen from Fig. 1).

The question can be posed: are these systems unique objects, or are there similar cool semi-detached systems which would smoothly bridge the gap in physical parameters between these pairs and hotter semi-detached binaries?

It is also convenient, after Popper (1980), to distinguish a group of hot semi-detached binaries, containing 33 catalogued systems. The hotter component in these systems can be as late as B4 and the spectral type of the cool component is B1 to A5.

For the remaining so-called classical algols the spectral type of the secondary is F0-K5 and differs from the primary's one by more than one spectral class. Four exceptions, having too similar spectral types of the components, are RW Cap, BR Cyg, WW And, AW Peg. There are some indications in the catalogues of Surkova \& Svechnikov (2005) and Budding et al. (2004) that the spectral type of the secondary in these systems is later than indicated in the GCVS.

Contact systems (C). As our analysis shows, spectra of latetype systems range from A3 to G5 (CWA) and from F5 to $\mathrm{K} 0(\mathrm{CWW})$. The spectral type of $\mathrm{CB}$ systems is B0-K0, while the spectral type of CE systems range from mid-O to B7.

The spectral type of the secondaries of CW and CE systems typically do not differ from the primary's one by more than a half of a spectral class, but spectra of $\mathrm{CB}$ secondaries exceed this limit.

Luminosity classes. For 551 systems in the catalogue, the luminosity class of at least one component is known, in particular for 327 systems with known evolutionary classes.

The luminosity class of both components in DM, DR, C and some $\mathrm{S}$ systems is IV or V. Four exceptions, IT Nor (CB), SV Cen (CE), LY Aur (CE) and V348 Car (CB) contain a giant. The extremely long periods of the two latter systems were
Table 2. Evolutionary classification: sufficient and necessary conditions.

\begin{tabular}{|c|c|}
\hline Class & Condition \\
\hline \multicolumn{2}{|r|}{$\begin{array}{l}\text { Sufficient conditions } \\
\end{array}$} \\
\hline $\mathrm{D}$ & $\mid$ (MinII-MinI)-0.5| $>0.025$ or $\quad$ DI-DII $>+0.01$ \\
\hline DG & $P>40^{\mathrm{d}}$ \\
\hline S & $A_{1}>3.7$ \\
\hline $\mathrm{C}$ & EW morphological type \\
\hline CWW & $P<0.26$ or $A_{2}>0.86$ \\
\hline \multicolumn{2}{|r|}{ Necessary conditions } \\
\hline $\mathrm{D}$ & $A_{2} \leq A_{2}^{R}$, see Eq. $(2)$ \\
\hline DM & $A_{1} \leq A_{1}^{M}+\delta A_{1}$, see Eq. (6) \\
\hline DR & EA morphological type \\
\hline S & $A_{2} \leq A_{2}^{R}+0 .^{m} 2$, see Eq. (2) \\
\hline CBV & Period does not increase \\
\hline $\mathrm{CE}$ & Period does not decrease \\
\hline
\end{tabular}

discussed above. It is advisable to distinguish these four systems in a separate group of giant contact binaries.

Only one (or no) component of S systems can be a giant (or larger). However, both components are giants in the cool semidetached systems AR Mon and RZ Cnc, discussed above.

The luminosity class of at least one component is I-II-III for DG systems.

\subsection{Summary of the rules}

We have investigated the distribution of the catalogued systems in some observational planes and extracted from them a set of rules that allows the classification of a given system.

The distribution of systems along the depth of minima is given in Fig. 1. Limiting amplitudes and periods for the catalogued systems of different classes are presented in Table 1. The results on the phase of secondary minimum and duration of eclipses are summarised in Sect. 3.5. Data on spectra of the catalogued systems are presented in Fig. 2. The relations between evolutionary class and non-numerical parameters (light curve morphological type and period change) are summarised in Sects. 3.2 and 3.4, respectively.

Table 2 lists sufficient and necessary conditions for a system to belong to a particular evolutionary class.

The rules developed here were used to design a procedure for eclipsing binary classification. The procedure assigns an evolutionary class to a system, based on its available observational parameters. One of the following classes can be assigned: D (detached system of unknown sub-class), DM, DR, DG, S, C, CB, $\mathrm{CBV}, \mathrm{CBF}, \mathrm{CE}, \mathrm{CW}, \mathrm{CWA}, \mathrm{CWW}$. If, according to the result of the classification, a system can belong to more than one of the listed classes, the class of the system is considered to remain unknown. However, if a system could belong to both DM and DR, it was classified as $\mathrm{D}$, etc.

\section{Application of the procedure}

In order to estimate the effectiveness of the procedure, it was tested on the set of 1029 systems with known classifiers. Altogether 475 systems (46\%) were classified, the class of others remained unknown. In 189 cases a less accurate classifier was assigned to a system (e.g., some CWW systems were classified as CW). However, for 19 systems a classifier was made more accurate (e.g., a CB system was classified as CBV). In general, 113 of 194 (58\%) D systems, 79 of 437 (18\%) S systems and 283 of 398 (71\%) C systems were classified correctly. 
Table 3. Results of classification.

\begin{tabular}{|c|c|c|c|c|c|c|c|c|c|c|c|c|c|c|c|c|}
\hline Survey & (1) & (2) & (3) & $\overline{\mathrm{D}}$ & $\overline{\mathrm{DM}}$ & $\overline{\mathrm{DR}}$ & $\overline{\mathrm{DG}}$ & $\bar{S}$ & $\overline{C \mathrm{C}}$ & $\overline{\mathrm{CB}}$ & $\overline{\mathrm{CBF}}$ & $\overline{\mathrm{CBV}}$ & $\overline{\mathrm{CE}}$ & $\overline{\mathrm{CW}}$ & $\overline{\text { CWA }}$ & CWW \\
\hline \multirow[t]{2}{*}{ CEV } & 5301 & 13 & 86 & 48 & 58 & 1 & 81 & 199 & 521 & 33 & 3 & & 7 & 15 & & 24 \\
\hline & & & $2 \%$ & $1 \%$ & $1 \%$ & & $2 \%$ & $4 \%$ & $10 \%$ & $1 \%$ & & & & & & \\
\hline \multirow[t]{2}{*}{ Wood-5 } & 3564 & 10 & 76 & 49 & 58 & 2 & 84 & 166 & 43 & 9 & 2 & & & 25 & 1 & 23 \\
\hline & & & $2 \%$ & $1 \%$ & $2 \%$ & & $2 \%$ & $5 \%$ & $1 \%$ & & & & & $1 \%$ & & $1 \%$ \\
\hline \multirow[t]{2}{*}{ OGLE-LMC } & 2681 & 6 & 266 & 299 & 141 & & 119 & 69 & 40 & 8 & & & 25 & 2 & & 3 \\
\hline & & & $10 \%$ & $11 \%$ & $5 \%$ & & $4 \%$ & $3 \%$ & $1 \%$ & & & & $1 \%$ & & & \\
\hline \multirow[t]{2}{*}{ OGLE-SMC } & 1404 & 6 & 65 & 202 & 53 & & 133 & 16 & 104 & 6 & & & 23 & 1 & & 1 \\
\hline & & & $5 \%$ & $14 \%$ & $4 \%$ & & $9 \%$ & $1 \%$ & $7 \%$ & & & & $2 \%$ & & & \\
\hline \multirow[t]{2}{*}{ MACHO } & 6143 & 3 & 24 & 656 & 197 & & 272 & 24 & & & & & & 11 & & 14 \\
\hline & & & & $11 \%$ & $3 \%$ & & $4 \%$ & & & & & & & & & \\
\hline \multirow[t]{2}{*}{ ASAS3-ED } & 3878 & 2 & 40 & 1 & & & 113 & 29 & & & & & & 12 & & 11 \\
\hline & & & $1 \%$ & & & & $3 \%$ & $1 \%$ & & & & & & & & \\
\hline \multirow[t]{2}{*}{ ASAS3-ESD } & 6219 & 2 & 337 & 3 & & & 280 & 30 & & & & & & 208 & & 127 \\
\hline & & & $5 \%$ & & & & $5 \%$ & & & & & & & $3 \%$ & & $2 \%$ \\
\hline \multirow[t]{2}{*}{ ASAS3-EC } & 5384 & 2 & 35 & 2 & & & 137 & 14 & & & & & & 301 & & 152 \\
\hline & & & $1 \%$ & & & & $3 \%$ & & & & & & & $6 \%$ & & $3 \%$ \\
\hline
\end{tabular}

(1) Number of stars in survey, (2) Number of used parameters, (3) Number of unclassified systems.

None from the 22 DR systems and 72 CWA systems were classified correctly; all of them were classified as D and $\mathrm{C} / \mathrm{CW}$ systems, correspondingly (or class remained unknown).

The procedure was then applied to the set of 5301 systems from the Malkov et al. (2005) catalogue of eclipsing variables with unknown classifiers. The results are summarized in the first row (CEV) of Table 3.

Unclassified systems. According to the results of the classification, 86 systems in the catalogue belong to none of the listed classes. There is a number of reasons for a system to remain unclassified.

1. A system can belong to a relatively rare evolutionary class that is not considered in the present paper and cannot be determined by the procedure. Examples are X-ray binaries V1341 Cyg and V1727 Cyg, cataclysmic variables RW Tri, AN UMa and LX Ser, polar EF Eri, symbiotic stars V1329 Cyg and AR Pav and some others.

2. For an eclipsing binary with a very shallow (and hence nonobserved) secondary minimum the next primary minimum can be erroneously considered to be the secondary one. In this case the catalogued period will be twice as long as the real one, and the catalogued depths of minima will be equal. Assuming that unclassified systems RU Ind and AP Aps (with catalogued values $A_{1}=A_{2}$ ) belong to such a "missed secondary minimum" category and correcting their data correspondingly $\left(A_{2}=0, P=0.5 P_{\text {catalogued }}\right)$, we found them to be detached or semi-detached systems.

3. For a system with equal depths of minima, a shallow minimum between them can be erroneously assumed to exist. That shallow minimum will be considered as a secondary one, consequently, the catalogued period will be half as long as the real one and the catalogued depth of secondary minimum will be equal to or near zero. Applying this assumption to an unclassified HV TrA and correcting their catalogued data correspondingly $\left(A_{2}=A_{1}, P=2 P_{\text {catalogued }}\right)$, it was found to be a DM system.

4. Stars with constant light can be seen in the GCVS, e.g., according to Hoffmann (1977) the variability of NW Aur is not real. This system was also unclassified by our procedure.

5. There may be out of date or erroneous data in the GCVS.
Other lists of eclipsing binaries. The procedure was applied to other catalogues and lists of eclipsing binaries. Large enough lists, containing thousands of systems with at least two known parameters, were selected. They are (known parameters are given in square brackets):

- A finding list for observers of interacting binary stars, fifth edition (Wood et al. 1980), [all parameters except morphological type of the light curve, DII and MinII-MinI];

- OGLE Catalog of eclipsing binary stars from the LMC (Wyrzykowski et al. 2003) and OGLE Catalog of eclipsing binary stars in the SMC (Wyrzykowski et al. 2004), $\left[A_{1}, A_{2}\right.$, $\mathrm{dA}$, morphological type of the light curve, $P$, MinII-MinI];

- MACHO list of eclipsing variables (Faccioli 2006, private communication), [ $P, A_{1}$, MinII-MinI];

- ASAS-3 Catalogue of variable stars, including ED (detached), ESD (semi-detached) and EC (contact) eclipsing variables (Pojmanski et al. 2005), $\left[P, A_{1}\right]$.

The results of the classification are summarized in Table 3. The lack of systems of some classes in the ASAS-3 survey is because DG systems have the largest $P, S$ systems have the largest $A_{1}$, CW systems have the smallest $\mathrm{P}$. To distinguish systems of other classes one usually needs to know values of other parameters, thus, the classes of these systems mostly remained unknown.

According to our results ASAS-3 catalogues of detached (ED), semi-detached (ESD) and contact (EC) eclipsing variables contain at least $1.3 \%, 9.9 \%$ and $2.8 \%$ of misclassified systems, respectively.

Lists of classified stars will be provided upon request.

\section{Conclusions}

The previously compiled catalogue of 6330 eclipsing binaries (Malkov et al. 2005), representing the largest list of eclipsing binaries classified from observations, has been used to develop the most comprehensive set of rules for the classification of eclipsing binaries to date.

We have investigated the distribution of the catalogued systems in various observational planes and extracted from them a number of rules that allows the classification of a given system based on a set of observational parameters, even if the set is incomplete. 
A few exceptions to these rules are: TX Gem (DM), ZZ Cep (DM), UZ Oct (CWA), CV Cyg (CWW). They require further study.

Three small groups of eclipsing binaries were distinguished, namely, inversed DR systems (RT Lac, Z Her), cool S systems (RZ Cnc, AR Mon, AD Cap, RV Lib) and giant C systems (IT Nor, SV Cen, LY Aur, V348 Car). They also do not satisfy the rules.

The developed procedure was applied to large catalogues of eclipsing variables and lists of eclipsing variables obtained as by-products of microlensing surveys. The results are summarized in Table 3, and lists of classified stars are available upon request. Altogether 5291 systems are classified for the first time. The classified systems can be used for the determination of the astrophysical parameters of their components.

The analysis also shows that the procedure can indicate errors in catalogued data and separate marginal classes of systems.

The procedure can be also applied to data to be generated from future ground-based and space observatories (GAIA, COROT).

Acknowledgements. We thank Nikolaj Samus for his very useful comments on the GCVS, Annemarie Bridges for her careful reading and constructive comments and Pavlos Protopapas and Lorenzo Faccioli for providing us with recent MACHO data. We thank our referee for helpful comments and suggestions. This work has been partially supported by the Spanish Ministerio de Ciencia y Tecnologia under contracts ESP 2001-4531-PE and ESP 2003-04352, by Russian Foundation for Fundamental Research grants 05-02-16123 and 06-07-89188, and by "The Support of Leading Scientific Schools" Program. This study was partly done while OM occupied a post of chercheur associé CNRS at the Université de Franche-Comté. This research has made use of the SIMBAD database, operated at the Centre de Données astronomiques de Strasbourg and of NASA's Astrophysics Data System Bibliographic Services.

\section{References}

Antokhina, E. A., Kreiner, J. M., Tremko, J., \& Cherepashchuk, A. M. 1987, PAZh, 13, 417

Bessell, M. S. 2000, PASP, 112, 961
Binnendijk, L. 1977, Vistas Astron., 21, 359

Budding, E., Erdem, A., Çiçek, C., et al. 2004, A\&A, 417, 263

Cakirli, O., Ibanoglu, C., Djurasevic, G., et al. 2003, A\&A, 405, 733

Cester, B., Fedel, B., Giuricin, G., Mardirossian, F., \& Mezzetti, M. 1978, A\&ASS, 33, 91

Giuricin, G., Mardirossian, F., \& Mezzetti, M. 1983, A\&ASS, 54, 211

Hilditch, R. W., \& Evans, T. L. 1985, MNRAS, 213, 75

Hilditch, R. W., Collier Cameron, A., Hill, G., Bell, S. A., \& Harries, T. J. 1997, MNRAS, 291, 749

Hoffmann, M. 1977, A\&A, 61, 145

Kholopov, P. N., Samus, N. N., Frolov, M. S., et al. 1985-1988, General Catalogue of Variable Stars, 4th edn., Vols. I-III, ed. O. V. Durlevich, M. S. Frolov, E. V. Kazarovets, \& N. N. Samus (Moscow: Nauka); 1994, The list of errors in the GCVS, 4th edn. I. Vols. I-III, Bull. Inf. CDS, 45, 19. See also http://www.sai.msu.su/groups/cluster/gcvs/gcvs/ for living edition

Lapasset, E., \& Sistero, R. F. 1984, A\&A, 130, 97

Malkov, O., Oblak, E., Snegireva, E. A., \& Torra, J. 2005, A\&A, 446, 785

Marques, J. P., Fernandes, J., \& Monteiro, M. J. P. F. G. 2004, A\&A, 422, 239

Nelson, C. A., \& Eggleton, P. P. 2001, ApJ, 552, 664

Olson, E. C. 1989, AJ, 98, 1002

Pojmanski, G., Pilecki, B., \& Szczygiel, D. 2005, AcA, 55, 275

Popper, D. M. 1976, ApJ, 208, 142

Popper, D. M. 1980, ARA\&A, 18, 115

Popper, D. M., \& Ulrich, R. K. 1977, ApJ, 212, L131

Pribulla, T., Kreiner, J. M., \& Tremko, J. 2003, Contributions of the Astronomical Observatory Skalnate Pleso, 33, 38

Rucinski, S. M., \& Maceroni, C. 2001, AJ, 121, 254

Shaw, J. S. 1994, Mem. S. A. It., 65, 1

Stickland, D. J., Koch, R. H., Pachoulakis, I., \& Pfeiffer, R. J. 1994, The Observatory, 114, 107

Surkova, L. P., \& Svechnikov, M. A. 2005, Catalogue of semi-detached eclipsing binaries. VizieR on-line data catalog V/115

Svechnikov, M. A. 1969, Catalogue of orbital elements, masses and luminosities of close binary stars, Sverdlovsk, Publ. Ural University, Ser. Astronomia, issue, $5, \mathrm{~N} 88$

Svechnikov, M. A., \& Snezhko, L. I. 1974, in Evidences of non-stationarity and stellar evolution, ed. A. A. Boyarchuk, \& Yu. N. Efremov (Moscow: Nauka), 181

Vinko, J., Hegedus, T., \& Hendry, P. D. 1996, MNRAS, 280, 489

Wood, F. B., Oliver, J. P., Florkowski, D. R., \& Koch, R. H. 1980, Publ. Department of Astronomy, University of Florida, Vol. I = Publ. Univ. of Pennsylvania, Astronomical Series, Vol. XII

Wyrzykowski, L., Udalski, A., Kubiak, M., et al. 2003, AcA, 53, 1

Wyrzykowski, L., Udalski, A., Kubiak, M., et al. 2004, AcA, 54, 1 